\title{
Sulfated polymannuroguluronate inhibits Tat-induced SLK cell adhesion via a novel binding site, a KKR spatial triad
}

\author{
Yan-lin $\mathrm{WU}^{1, \#}$, Jing $\mathrm{Al}^{2, \#}$, Jing-ming $\mathrm{ZHAO}^{3}$, Bing XIONG${ }^{4}$, Xiao-jie XIN² , Mei-yu GENG ${ }^{2}$, Xian-liang XIN ${ }^{2, *}$, Han-dong JIANG ${ }^{3, *}$ \\ ${ }^{1}$ Department of Pharmacology and Glycobiology, School of Medicine and Pharmacy, Ocean University of China, Qingdao 266003, \\ China; ${ }^{2}$ Division of Anti-tumor Pharmacology, State Key Laboratory of Drug Research, Shanghai Institute of Materia Medica, Chinese \\ Academy of Sciences, Shanghai 201203, China; ${ }^{3}$ Division of Respiratory Medicine, Affiliated Hospital of Medical College, Qingdao \\ University, Qingdao 266003, China; ${ }^{4}$ Department of Medicinal Chemistry, State Key Laboratory of Drug Research, Shanghai Insti- \\ tute of Materia Medica, Chinese Academy of Sciences, Shanghai 201203, China
}

Aim: Sulfated polymannuroguluronate (SPMG), a candidate anti-AIDS drug, inhibited HIV replication and interfered with HIV entry into host T lymphocytes. SPMG has high binding affinity for the transactivating factor of the HIV-1 virus (Tat) via its basic domain. However, deletion or substitution of the basic domain affected, but did not completely eliminated Tat-SPMG interactions. Here, we sought to identify other SPMG binding sites in addition to the basic domain.

Methods: The potential SPMG binding sites were determined using molecular simulation and a surface plasmon resonance (SPR) based competitive inhibition assay. The effect of SPMG on Tat induced adhesion was evaluated using a cell adhesion assay.

Results: The KKR domain, a novel high-affinity heparin binding site, was identified, which consisted of a triad of Lys12, Lys41, and Arg78. The KKR domain, spatially enclosed SPMG binding site on Tat, functions as another binding domain for SPMG. Further functional evaluation demonstrated that SPMG inhibits Tat-mediated SLK cell adhesion by directly binding to the KKR region.

Conclusion: The KKR domain is a novel high-affinity binding domain for SPMG. Our findings provide important new insights into the molecular mechanisms of SPMG and a potential therapeutic intervention for Tat-induced cell adhesion.

Keywords: sulfated polymannuroguluronate; transactivating factor of the HIV-1 virus (Tat); binding site; Kaposi's sarcoma; heparin; mutation; HIV-1

Acta Pharmacologica Sinica (2011) 32: 647-654; doi: 10.1038/aps.2011.2; published online 18 Apr 2011

\section{Introduction}

Kaposi's sarcoma (KS) is the most common tumor in human immunodeficiency virus (HIV)-infected patients and is a leading cause of mortality and morbidity in acquired immune deficiency syndrome (AIDS) ${ }^{[1-3]}$. KS is much more frequent and aggressive in the setting of HIV-1 infection, suggesting that HIV itself, or molecules produced during HIV infection, might play important roles in KS development and progression ${ }^{[4]}$. The transactivating factor of the HIV-1 virus (Tat), a polypeptide of 86-102 amino acids released from HIV-1 infected cells, contributes directly to KS pathogenesis ${ }^{[5]}$. In addition to the transactivation of HIV-1 gene expression, Tat can promote KS

\footnotetext{
\# These two authors contributed equally to this work.

* To whom correspondence should be addressed.

E-mail jianghd@163.com (Han-dong JIANG); xinxianliang@hotmail.com (Xian-liang XIN)

Received 2010-11-12 Accepted 2011-01-09
}

cell adhesion through binding to integrins ${ }^{[6,7]}$. Therefore, the inhibition of Tat activity could be a useful way to inhibit the development and progression of KS in AIDS patients.

Tat-driven activities depend precisely on its interaction with cell surface heparan sulfate (HS) and heparin through their negatively charged sulfate groups ${ }^{[8]}$. Conversely, free heparin inhibits the uptake of intracellular Tat and affects the cell surface interaction mediated by $\mathrm{Tat}^{[9-11]}$. The basic domain constituted by a stretch of repeated Arg and Lys residues has long been recognized as the sole contributor to this interaction, which is mediated by its heparin binding properties ${ }^{[9,11-13]}$. However, our previous studies have challenged this notion by identifying a novel heparin-binding KKR domain (Lys12, Lys41, and Arg78) that helps facilitate Tat-driven $\beta 1$ integrin activation and subsequent adhesion in a heparan sulfate proteoglycan (HSPG)-dependent manner ${ }^{[14]}$. These important insights into the binding profile between heparin and Tat will provide a more precise understanding of the comprehensive 
roles of Tat and aid in the design of new therapeutic agents. Moreover, growing evidence shows that heparin and many heparin analogs represent potent extracellular Tat antagonists of possible therapeutic value ${ }^{[10,15]}$.

Sulfated polymannuroguluronate (SPMG), a new heparinlike sulfated polysaccharide extracted from brown algae, is rich in 1,4-linked $\beta$-D-mannuronate, with an average of 1.5 sulfates and 1.0 carboxyl groups per sugar residue and an average molecular weight of $10 \mathrm{kDa}$. SPMG has entered Phase II clinical trials in China, making it the first marine sulfated polysaccharide with the potential to be approved as an antiAIDS drug. Our previous in vitro and in vivo studies demonstrated that SPMG inhibited HIV replication and interfered with HIV entry into host T lymphocytes. We further demonstrated that Tat exhibited a high binding affinity for SPMG via its basic domain, thereby inhibiting Tat-induced AIDS-KS ${ }^{[16,17]}$. We also found that substitution of the basic domain affected, but failed to completely eliminate, Tat-SPMG interactions. These results led us to hypothesize that there might exist other SPMG binding sites in addition to the basic domain. In this article, using molecular simulation combined with a surface plasmon resonance (SPR)-based competitive inhibition assay, we found that the KKR domain, a newly identified highaffinity heparin binding site ${ }^{[14]}$, functioned as another binding domain for SPMG. Further functional evaluation demonstrated that SPMG inhibited Tat-mediated SLK cell adhesion by directly binding to the KKR region.

\section{Materials and methods Materials}

SPMG, depolymerized by acidic hydrolysis, and then sulfatation $\left(M_{\mathrm{w}}\right.$ about $\left.8000 \mathrm{Da}\right)$, was provided by the Marine Drug and Food Institute (Ocean University of China, Qingdao, China). Heparin, HS, chondroitin sulfates A (CSA) and C (CSC), and hyaluronic acid (HA) were obtained from Sigma (St Louis, MO, USA). Anti-GST antibody (sc-459) was purchased from Santa Cruz Biotechnology, Inc (Santa Cruz, CA, USA).

\section{Cell culture}

SLK cells (derived from an endothelial-origin Kaposi's sarcoma) were kindly provided by the NIH AIDS Research Division. The cells were maintained in RPMI-1640 medium supplemented with 10\% heat-inactivated fetal calf serum (FCS, Gibco Gaithersburg, MD, USA), 2 mmol/L L-glutamine, 100 $\mathrm{U} / \mathrm{mL}$ penicillin, and $100 \mu \mathrm{g} / \mathrm{mL}$ streptomycin at $37^{\circ} \mathrm{C}$ in a humidified incubator with $5 \% \mathrm{CO}_{2}$.

\section{Computational docking modeling}

The amino acid sequence of human T-lymphotropic virus 3 (Tat-III protein) was retrieved from the appropriate NCBI protein database (GI: 328765) and used to search for homologs in the Protein Data Bank (http://www.rcsb.org). Through a BLASTP search, it was found that the NMR structure of Tat-I (PDB entry: 1JFW) was the most homologous protein (83/86 aa, $96 \%$ identity ${ }^{[18]}$. Therefore, the structure of Tat-I was used

as the template to create a 3D model of Tat-III using the MODELLER program ${ }^{[19]}$. Ten models were generated, and these 3D structures were optimized with a conjugate gradient minimization scheme followed by a restrained annealing molecular dynamics simulation. The model with the lowest value for the objective function was selected as the most representative TatIII model for further study.

Structure-based analysis of our homology model of TatIII was performed to search for SPMG binding sites using the newly developed AutoDock Vina program ${ }^{[20]}$. This program is two orders of magnitude faster than AutoDock4 and enabled us to search for binding sites over the whole surface of Tat-III. Additionally, it is based on the fast iterated local search global optimization of protein-ligand interactions, so the search time increases linearly with atom number. The input PDBQT files of Tat-III and three ligands (2-, 4-, and 8-SPMG) were prepared with AutoDockTools, and the docking operation algorithm was performed in a consistent manner. A large box encompassing the entire Tat-III structure, with dimensions $48 \AA \times 48$ $\AA \times 42 \AA$, was generated to take all possible SPMG binding sites into account. Then, the Vina program was applied to account for protein-ligand interactions using a newly developed scoring function that includes the terms gauss type steric interaction, repulsion interaction, hydrogen bond, and torsional free energy. Through large scale validation, the Vina program can significantly improve the accuracy of binding mode predictions ${ }^{[20]}$. Finally, the evaluation with the lowest binding energy was used to analyze ligand pose, and the interaction model of SPMG/Tat-III was illustrated with the program Pymol based on the docked complex structure ${ }^{[21]}$.

\section{Preparation of Tat and its mutants}

Plasmid pGEX-2T (Amersham Pharmacia, Uppsala, Sweden) and pGST-Tat were kindly provided by Prof M GIACCA and M PRESTAI (International Centre for Genetic Engineering and Biotechnology, Trieste, Italy). Tat mutants were constructed using site-directed mutagenesis, yielding a series of GST-Tat mutants (Table 1).

Recombinant wild type HIV-1 Tat and the different Tat mutants were expressed in Escherichia coli as glutathione $S$-transferase (GST) fusion proteins and purified to homoge-

Table 1. Summary of mutated amino acids in the set of GST-Tat mutants.

Mutant name

GST-Tat $_{(\mathrm{G} 48-\mathrm{R} 57) \mathrm{A}}$

GST-Tat $_{\mathrm{K}(12,41) \mathrm{A} / \mathrm{R} 78 \mathrm{~A}}$

GST-Tat $_{\text {K12A }}$

GST-Tat $_{\text {K41A }}$

GST-Tat $_{\text {R78A }}$

GST-Tat $_{\mathrm{K}(12,41) \mathrm{A}}$

GST-Tat $_{\text {K12A/R78A }}$

GST-Tat $_{\text {K41A/R78A }}$

GST-Tat $_{(G 48-R 57) A / K(12,41) A / R 78 A}$
Amino acids change

Amino acids 48-57 (basic domain) $\rightarrow$ Ala

Lys12, Lys41, Arg78 $\rightarrow$ Ala

Lys12 $\rightarrow$ Ala

Lys $41 \rightarrow$ Ala

Arg78 $\rightarrow$ Ala

Lys12, Lys41 $\rightarrow$ Ala

Lys12, Arg78 $\rightarrow$ Ala

Lys41, Arg78 $\rightarrow$ Ala

Amino acids 48-57, Lys12, Lys41, Arg78

$\rightarrow$ Ala 
neity from bacterial lysates by glutathione-sepharose affinity chromatography (Amersham Pharmacia) as described previously ${ }^{[14]}$. The purity and integrity of the protein product were routinely checked by SDS-polyacrylamide gel electrophoresis and $0.25 \%$ Coomassie brilliant blue R-250 staining. The identification of mutant products was further carried out by MS-MS spectral analysis. In addition, the similarity of the secondary structure of the mutated products to the native Tat protein was predicted using a position-specific scoring method ${ }^{[14]}$. The purified proteins were stored in aliquots at $-80^{\circ} \mathrm{C}$ until use $e^{[9]}$.

\section{Surface plasmon resonance assay}

To characterize Tat binding with SPMG, kinetic properties and competitive inhibition were examined using a surface plasmon resonance assay (SPR, BIAcore X, Uppsala, Sweden). For this purpose, SPMG was immobilized to a CM5 sensor chip according to the established method ${ }^{[22,23]}$. The immobilization procedure was carried out at $25^{\circ} \mathrm{C}$ and at a constant flow rate of $5 \mu \mathrm{L} / \mathrm{min}$ in HBS-EP [0.01 mol/L HEPES, pH 7.4, $0.15 \mathrm{~mol} / \mathrm{L} \mathrm{NaCl}, 3 \mathrm{mmol} / \mathrm{L}$ EDTA, and $0.005 \%$ polysorbate $20(v / v)]$. To assess the real-time binding capacity, $50 \mu \mathrm{L}$ of GST-Tat or the relevant mutant was injected over the sensor chip surface with the immobilized SPMG, followed by 5 min of washing with HBS-EP buffer. The sensor chip surface was regenerated using $2 \mathrm{~mol} / \mathrm{L} \mathrm{NaCl}$. All binding experiments were performed at $25{ }^{\circ} \mathrm{C}$ with a constant flow rate of 15 $\mu \mathrm{L} / \mathrm{min}$ HBS-EP. To correct for nonspecific binding and bulk refractive index change, a blank channel (FC2) without SPMG was employed as a control for each experiment. Sensorgrams for all binding interactions were recorded in real time and analyzed after subtraction of channel blanks. Changes in mass due to the binding response were recorded as resonance units (RU). Binding kinetics and affinities were calculated using BIAcore software 3.1. Competitive inhibition experiments were conducted with the same protocol, except that a $50 \mu \mathrm{L}$ mixture of GST-Tat or the relevant mutant and inhibitors that had been preincubated for $3 \mathrm{~min}$ at $37^{\circ} \mathrm{C}$ was used.

\section{Cell adhesion assay}

To guarantee that plates were coated with the same amount of each tested protein, anti-GST antibody was adsorbed to polystyrene microplates (Costar, Cambridge, MA, USA) by overnight incubation at $4{ }^{\circ} \mathrm{C}$ in $100 \mathrm{mmol} / \mathrm{L}$ carbonate buffer $(\mathrm{pH}$ 9.6) at a concentration of $10 \mu \mathrm{g} / \mathrm{mL}$. The plates were subsequently washed three times with Tris-buffered saline+Tween 20 (TBS-T) and then filled with $1.25 \%$ BSA-PBS and incubated for $2 \mathrm{~h}$ at $37^{\circ} \mathrm{C}$. After three washes with TBS-T, $40 \mu \mathrm{g} / \mathrm{mL}$ of GST alone, GST-Tat or the relevant mutant Tat protein was added to the wells and incubated for $1 \mathrm{~h}$ at $37^{\circ} \mathrm{C}$. The plate was next washed three times with PBS-T. Then, $100 \mu \mathrm{L}$ of SLK cell suspension was added to each well $\left(3 \times 10^{5}\right.$ cells $/ \mathrm{mL}$ in RPMI-1640 with $10 \%$ FCS). After incubation for $1 \mathrm{~h}$ at $37{ }^{\circ} \mathrm{C}$ in a $5 \% \mathrm{CO}_{2}$ atmosphere, non-adherent cells were removed by careful aspiration and three washes with RPMI 1640. Adherent cells were fixed, stained, and quantified as previously described $^{[24]}$. A SPMG competition cell adhesion assay was conducted with the same procedure, except that SPMG at defined concentrations was added to each well. Each experiment was performed in triplicate and replicated three times.

\section{Statistical analysis}

Data were presented as means $\pm \mathrm{SD}$, and differences were considered significant when $P<0.05$ as determined by Student's $t$-tests.

\section{Results}

Lys12, Lys41, and Arg78 triad is predicted as a novel SPMG binding site

Computational modeling was first employed with the aim of identifying potential SPMG binding sites. The homology model of Tat protein (Tat-III) constructed on the basis of NMR data was used as the target structure, and several SPMG oligosaccharides including di-, tetra-, and octa-saccharides were selected as docking probes. In the disaccharide SPMG model, SPMG was noted to preferentially interact with a triad of basic residues (Lys12, Lys41, and Arg78 (KKR); Figure 1A-1C, highlighted in yellow in Figure 1C).

Modeling of interactions with tetra- and octa-saccharides revealed similar results; the positively charged KKR triad plays an important role in the molecular recognition of SPMG probes by producing a highly conserved interaction with Tat-III. It should be noted that the oligosaccharide chain begins to stretch toward the basic domain (green for comparison, Figure $1 \mathrm{D}$ and $1 \mathrm{E}$ ) as the probe length increases. As the length increases to a tetramer, a delicate interaction with residue Arg56 of the basic domain is established. The octamer follows the same path and approaches residue Arg53 (Figure 1D and $1 \mathrm{E}$ ). Ideally, SPMG, if long enough, is proposed to fully extend to encompass both the KKR region and the whole basic domain.

\section{The KKR domain is spatially enclosed to bind to SPMG}

To characterize the Tat KKR triad engagement with SPMG, a series of Tat mutants were expressed in E coli as GST-Tat fusion proteins, purified from bacterial lysates by glutathioneagarose affinity chromatography, and checked by SDS-PAGE.

First, the $\mathrm{EC}_{50}$ binding capacity was evaluated. For this evaluation, a series of GST-Tat solutions with a range of concentrations $(0.25,0.5,1,2$, or $4 \mu \mathrm{mol} / \mathrm{L})$ were injected over an SPMG-immobilized sensor chip surface. $\mathrm{RU}_{\max }$ values were generated using BIAcore evaluation 3.1 software, yielding an $\mathrm{EC}_{50}$ value of $1.5 \mu \mathrm{mol} / \mathrm{L}$ (data not shown); this value was used as a reference for subsequent experiments.

We next investigated whether KKR was an SPMG binding site, as predicted. A series of comparative studies were performed using all relevant mutants. We found that GSTTat binding to SPMG exhibited a multi-phasic binding profile (Figure 2A), indicating the existence of more than one SPMG binding site. Notably, mutation of the basic domain still produced monophasic binding, further supporting the notion that a binding site for SPMG exists in addition to the basic domain (Figure 2B). Accordingly, the mutation of Lys12, Lys41, and 
A
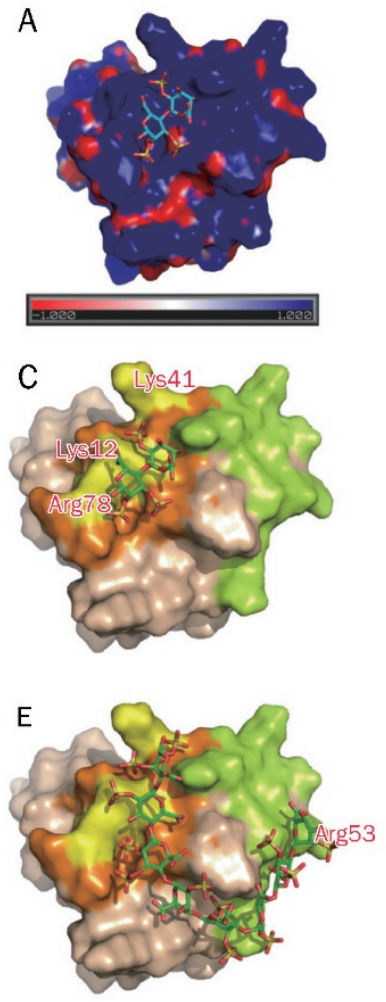

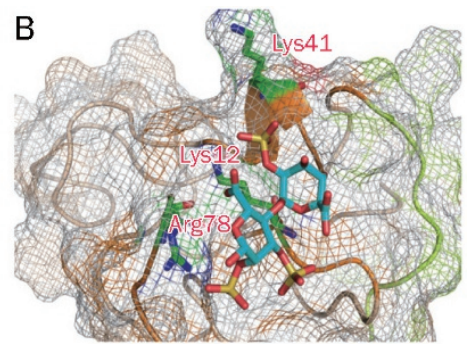

D

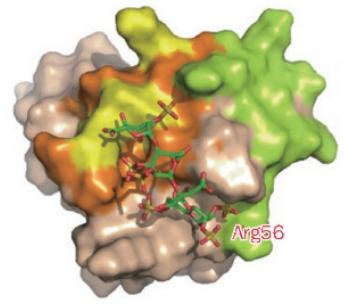

Figure 1. Computational docking model of HIV-Tat with SPMG-derived fragments. Predicted interactions between HIV-Tat and SPMG-derived fragments, including di- (A, B, and C), tetra- (D), and octa-saccharide (E) are indicated. Tat is represented by a surface and oligosaccharides by stick model. The Tat surface is colored by electrostatic potential in A. In $C-E$, it is wheat-colored, with the KKR region and basic domain highlighted in yellow and green, respectively. A close-up view of the binding interface between the di-saccharide and the KKR (Lys12, Lys41, and Arg78) region is shown in $B$, where Tat is shown in mesh form and the protein main chain is shown in cartoon style.

Arg78 to alanine [GST-Tat ${ }_{\mathrm{K}(12,41) \mathrm{A} / \mathrm{R} 78 \mathrm{~A}}$ ] displayed a markedly decreased binding potency for SPMG when compared to

wild-type GST-Tat (Figure 2C). Moreover, double-mutant GST-Tat $_{(\mathrm{G} 48-\mathrm{R} 57) \mathrm{A} / \mathrm{K}(12,41) \mathrm{A} / \mathrm{R} 78 \mathrm{~A}}$ led to complete abolishment of TatSPMG interactions (Figure 2C). These findings substantiate our hypothesis that Tat possesses another SPMG binding site, the KKR region.

We next clarified the contribution of the three basic residues as a whole and separately. Single, double, and triple mutations of the KKR region were constructed and their binding affinities $\left(K_{\mathrm{D}}\right)$ for immobilized SPMG were recorded and compared. $K_{\mathrm{D}}$ values were calculated using BIAcore evaluation 3.1 software. Mutation of a single residue (GST-Tat ${ }_{\mathrm{K} 12 \mathrm{~A}}$, GST-Tat $_{\text {K41A }}$, or GST-Tat ${ }_{\mathrm{R} 78 \mathrm{~A}}$ ) led to only a partial loss of SPMG binding affinity $\left(K_{\mathrm{D}} 0.112 \mathrm{nmol} / \mathrm{L}, 0.174 \mathrm{nmol} / \mathrm{L}\right.$, or 0.0729 $\mathrm{nmol} / \mathrm{L}$, respectively), with the Arg78 mutation (GST-Tat ${ }_{\mathrm{R} 78 \mathrm{~A}}$ ) influencing binding affinity the least (Table 2). Mutation of

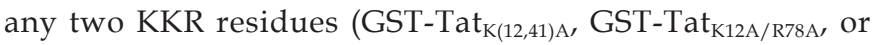

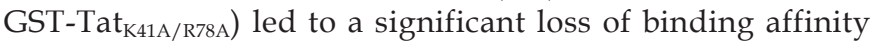
with correspondingly decreased $K_{\mathrm{D}}$ values $(1.04 \mathrm{nmol} / \mathrm{L}, 0.435$ $\mathrm{nmol} / \mathrm{L}$ and $0.205 \mathrm{nmol} / \mathrm{L}$, respectively), with the Lys12, Lys41 mutation (GST-Tat $\left.{ }_{\mathrm{K}(12,41) \mathrm{A}}\right)$ affecting binding the most (Table 2). Most significantly, concurrent mutation of all three residues produced a dramatic drop in SPMG binding affinity, with a resultant $K_{\mathrm{D}}$ value of $11 \mathrm{nmol} / \mathrm{L}$ (Table 2). These findings collectively indicated that Lys12, Lys41 and Arg78 cooperated as a whole to profoundly impact SPMG binding.

\section{The KKR triad binds specifically to SPMG in a high affinity manner}

We next sought to delineate the nature of the interaction between KKR and SPMG. We first assessed binding affinity. Various concentrations of GST-Tat, GST-Tat ${ }_{(\mathrm{G} 48-\mathrm{R} 57) \mathrm{A}}$, and GST-Tat $_{\mathrm{K}(12,41) \mathrm{A} / \mathrm{R} 78 \mathrm{~A}}$ were injected over an SPMG-immobilized sensor chip surface (Figure 3A-3C). We found that GST-Tat bound to SPMG $\left(K_{\mathrm{D}}=24.2 \mathrm{pmol} / \mathrm{L}\right)$ (Table 2$)$. Mutation of the basic domain (leaving the KKR domain intact and thus solely responsible for the strength of the Tat KKR-SPMG interaction) had little effect on binding affinity $\left(K_{\mathrm{D}}=26.5 \mathrm{pmol} / \mathrm{L}\right)$. Nota-
A

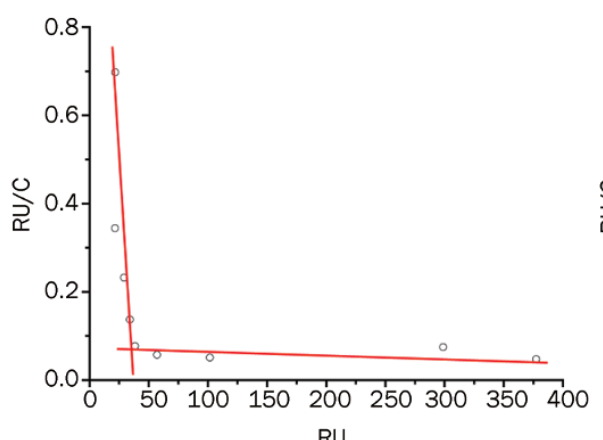

$\mathrm{RU}$
B

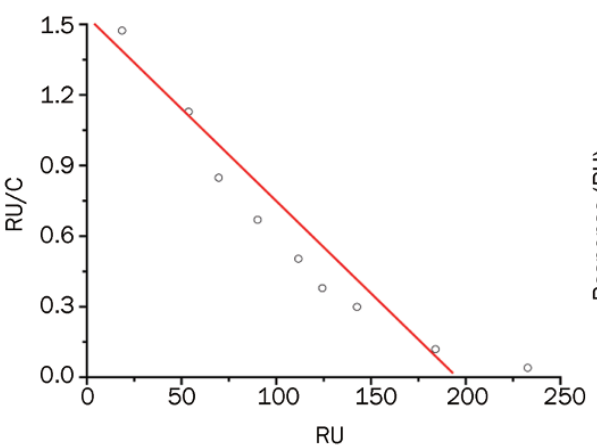

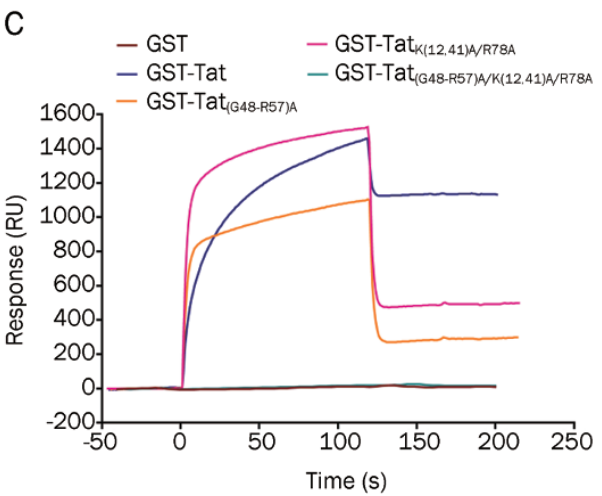

C

Figure 2. Identification of the Tat Lys12, Lys41, Arg78 (KKR) domain as a new SPMG binding site. (A, B) Scatchard plot analysis of GST-Tat (A) and GST-Tat $_{(\mathrm{G48-R57)A}}$ (B) with immobilized SPMG. A series of concentrations of GST-Tat or GST-Tat (G48-R57)A $_{\text {were }}$ injected over the SPMG-immobilized sensor chip surface. The binding potency/concentration (RU/C) was plotted versus RU. (C) Interaction of GST-Tat and its mutants with biotinylated SPMG immobilized on a CM5 sensor chip. GST-Tat or the appropriate mutants $(1.5 \mu \mathrm{mol} / \mathrm{L})$ were injected over the SPMG-immobilized sensor chip. The binding RU was recorded as binding potency. 
Table 2. The affinity parameters for binding of GST-Tat and its mutants to SPMG.

\begin{tabular}{|c|c|c|}
\hline Analyte & $K_{A}\left[1 /\left(m o l \cdot L^{-1}\right)\right]$ & $K_{\mathrm{D}}(\mathrm{mol} / \mathrm{L})$ \\
\hline GST-Tat & $4.13 \times 10^{10}$ & $2.42 \times 10^{-11}$ \\
\hline GST-Tat $_{(\mathrm{G} 48-\mathrm{R} 57) \mathrm{A}}$ & $3.77 \times 10^{10}$ & $2.65 \times 10^{-11}$ \\
\hline GST-Tat $_{\mathrm{K}(12,41) \mathrm{A} / \mathrm{R} 78 \mathrm{~A}}$ & $9.08 \times 10^{7}$ & $1.10 \times 10^{-8}$ \\
\hline GST-Tat $_{\mathrm{K} 12 \mathrm{~A}}$ & $8.93 \times 10^{9}$ & $1.12 \times 10^{-10}$ \\
\hline GST- Tat $_{\mathrm{K} 41 \mathrm{~A}}$ & $5.73 \times 10^{9}$ & $1.74 \times 10^{-10}$ \\
\hline GST- Tat $_{\text {R78A }}$ & $1.37 \times 10^{10}$ & $7.29 \times 10^{-11}$ \\
\hline GST- Tat $_{\mathrm{K}(12,41) \mathrm{A}}$ & $9.65 \times 10^{8}$ & $1.04 \times 10^{-9}$ \\
\hline GST-Tat $_{\text {K12A/R78A }}$ & $2.30 \times 10^{9}$ & $4.35 \times 10^{-10}$ \\
\hline GST- Tat $_{\text {K41A/R78A }}$ & $4.88 \times 10^{9}$ & $2.05 \times 10^{-10}$ \\
\hline
\end{tabular}

$K_{A}$ : equilibrium association constant; $K_{D}$ : equilibrium dissociation constant. Data shown are representative of three independent experiments with similar results.

bly, mutation of the KKR region caused a remarkable decrease in GST-Tat-SPMG binding $\left(K_{\mathrm{D}}=11 \mathrm{nmol} / \mathrm{L}\right)$, representing the $K_{\mathrm{D}}$ value of the basic domain in isolation. This $K_{\mathrm{D}}$ represents a 500 fold decrease in binding affinity compared with that of wild-type GST-Tat (Table 2). Based on this comparison, we conclude that the KKR spatial region dominates Tat-SPMG binding compared to the basic domain.

For further confirmation, we conducted a series of competitive inhibition assays. Wild-type GST-Tat or the appropriate Tat mutants $(1.5 \mu \mathrm{mol} / \mathrm{L})$ were injected over an SPMGimmobilized chip in the presence of varying concentrations of SPMG as a competitive inhibitor. Free SPMG was able to inhibit the binding of either GST-Tat or the corresponding mutant to immobilized SPMG in a dose-dependent manner (Figure 4A-4C). Notably, mutation of the basic domain (GSTTat $\left._{(\mathrm{G48}-\mathrm{R} 57 \mathrm{~A})}\right)$ produced an $\mathrm{IC}_{50}$ value $(1.803 \mu \mathrm{g} / \mathrm{mL})$ comparable to that of GST-Tat $(1.808 \mu \mathrm{g} / \mathrm{mL})$, while mutation of the KKR region $\left(\mathrm{GST}-\mathrm{Tat}_{\mathrm{K}(12,41) \mathrm{A} / \mathrm{R} 78 \mathrm{~A}}\right.$ ) yielded an $\mathrm{IC}_{50}$ value of 3.259 $\mu \mathrm{g} / \mathrm{mL}$, almost twice that of GST-Tat. These data further support the notion that the KKR spatial domain contributes dramatically to SPMG binding and represents an extremely highaffinity SPMG binding site.

We then characterized KKR binding specificity via the introduction of soluble glycosaminoglycans (GAGs). Both heparin and HS were able to compete for KKR binding to SPMG, yielding $\mathrm{IC}_{50}$ values of $0.633 \mu \mathrm{g} / \mathrm{mL}$ and $41.314 \mu \mathrm{g} / \mathrm{mL}$, respectively. By contrast, other GAGs including CSA, CSC, and HA, even at concentrations up to $125 \mu \mathrm{g} / \mathrm{mL}$, showed little or no inhibition of this interaction (Figure 4D). These findings suggest the binding specificity of the KKR region for SPMG is high.

Collectively, these data support the hypothesis that the KKR region of Tat, distinct from the well-recognized linearly contiguous basic domain, serves as a binding motif for SPMG that has extremely high affinity and is highly specific.

SPMG inhibits KKR-facilitated Tat-mediated SLK cell adhesion. Because the KKR region facilitates Tat-mediated SLK cell
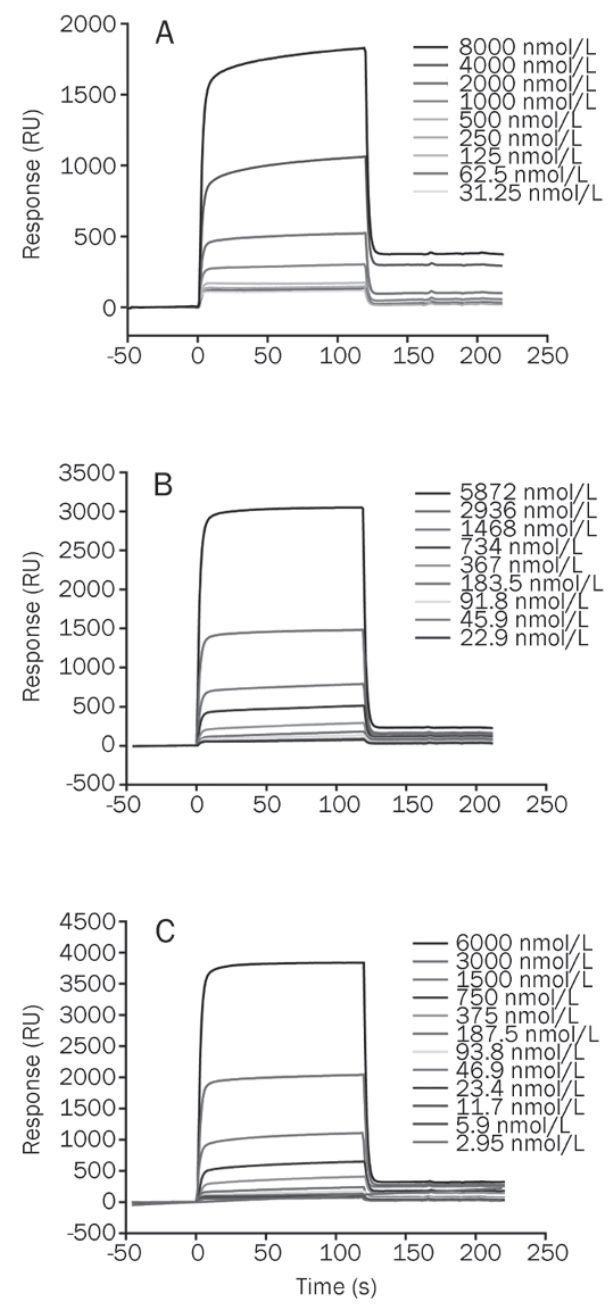

Figure 3. Characterization of the intrinsic properties of the Tat KKR SPMG binding domain. (A-C) Sensorgrams of GST-Tat and its mutants with immobilized SPMG. Serial concentrations of GST-Tat and its mutants were injected over the SPMG-immobilized sensor chip surface. The real time binding was recorded as response (RU) versus time. (A), (B) and (C)

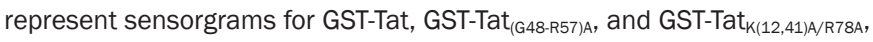
respectively.

adhesion in a cell surface HSPG-dependent manner ${ }^{[14]}$, we then examined the effects of SPMG on SLK cell adhesion. We found that both Tat and KKR-dominant Tat (GST-Tat $\left.{ }_{(\mathrm{G} 48-\mathrm{R} 57) \mathrm{A}}\right)$ significantly induced the adhesion of SLK cells, although the KKR-dominant Tat induced cell adhesion to a lower extent. Notably, $200 \mu \mathrm{g} / \mathrm{mL}$ of SPMG dramatically inhibited SLK adhesion (Figure 5). Together, these findings clearly support the idea that SPMG inhibits KKR region-facilitated SLK cell adhesion by directly binding to the KKR region.

\section{Discussion}

In the present study, we demonstrated that the triad of Lys12, Lys41, and Arg78 functioned as a crucial determinant of TatSPMG interaction in addition to the basic domain.

Using an SPR assay, a multiphasic binding mode between Tat and SPMG was observed. With mutation of the basic 

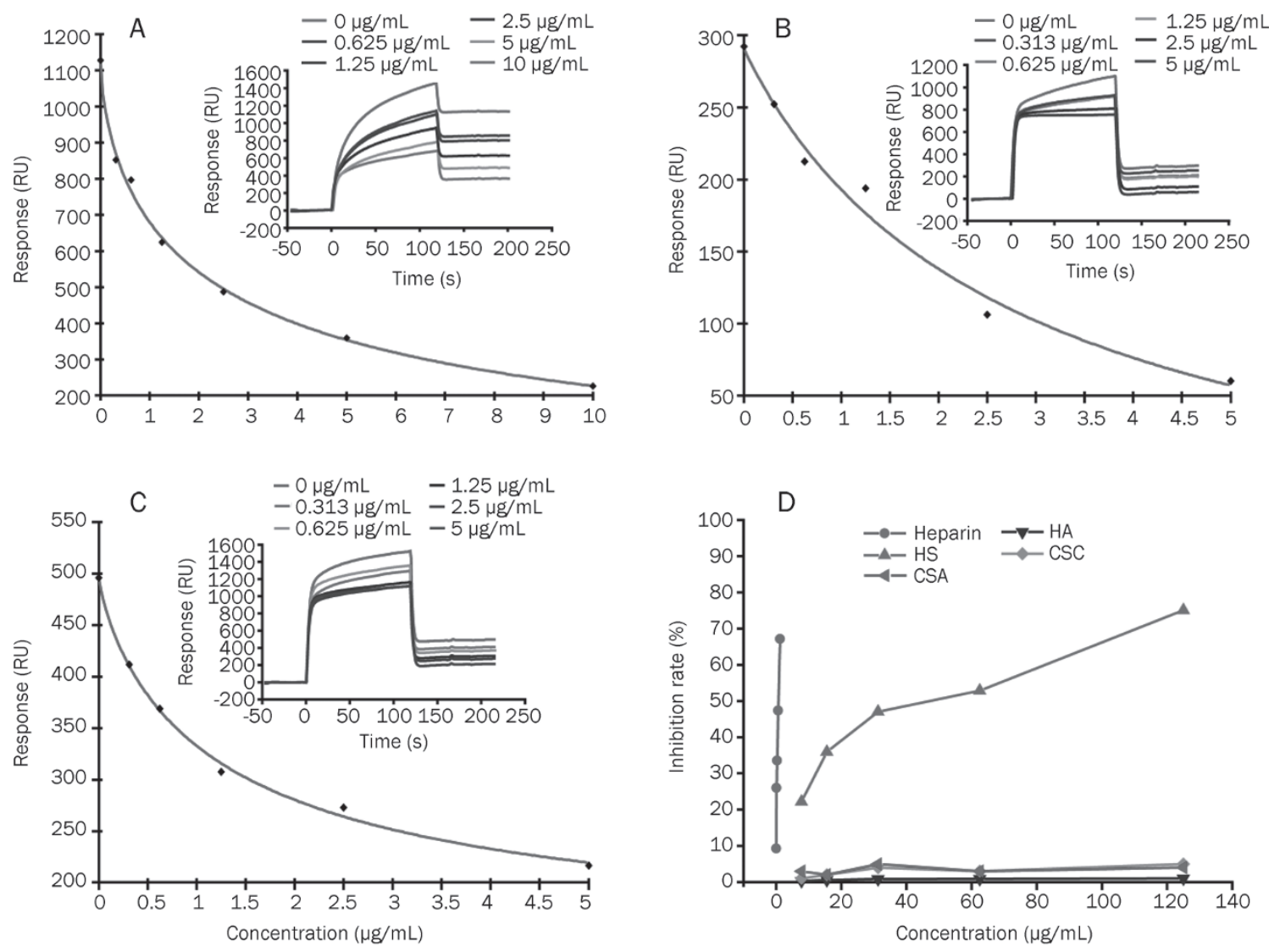

Figure 4. SPMG competitively inhibits binding of GST-Tat and its mutants with immobilized SPMG. GST-Tat (A), GST-Tat ${ }_{(\mathrm{G} 48-\mathrm{R} 57) \mathrm{A}}(\mathrm{B})$ and $_{\text {GST-Tat }} \mathrm{K}(12,41) \mathrm{A} / \mathrm{R} 78 \mathrm{~A}_{(\mathrm{C})}$ $(1.5 \mu \mathrm{mol} / \mathrm{L})$ alone or in the presence of serial concentrations of SPMG were injected over an SPMG immobilized chip surface. The responses (in RU) were plotted versus the concentration of SPMG. Insets show the overlay of sensorgrams showing the binding of GST-Tat and its mutants to immobilized SPMG in the presence of increasing concentrations of SPMG. (D) The inhibitory action of different glycosaminoglycans on GST-Tat ${ }_{\left(G 48-\text { R57) }^{-} \text {-SPMG }\right.}$ interaction. GST-Tat ${ }_{\mathrm{G} 48-\mathrm{R} 57 \mathrm{~A}}(1.5 \mu \mathrm{mol} / \mathrm{L})$ was preincubated with the indicated concentrations of free heparin, heparan sulfate (HS), chondroitin sulfates A (CSA), C (CSC), or hyaluronic acid (HA) and injected over a biotinylated SPMG surface.

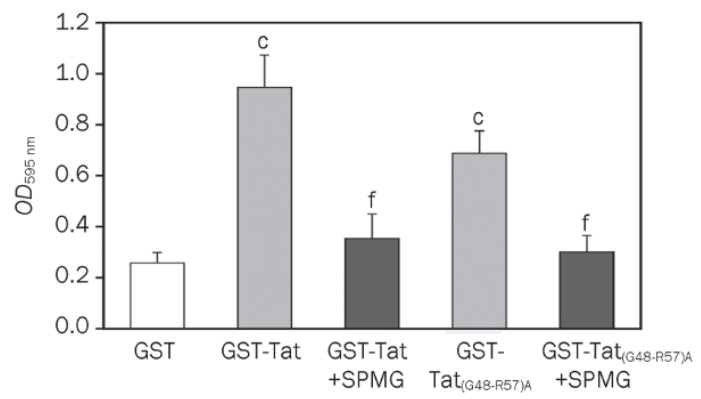

Figure 5. Inhibitory effects of SPMG on Tat-mediated SLK cell adhesion (n=3). ${ }^{\mathrm{c}} \mathrm{P}<0.01$ vs GST; ${ }^{\mathrm{f}} \mathrm{P}<0.01$ vs GST-Tat or GST-Tat $(\mathrm{G48-R57)A}$.

domain, a monophasic binding series became evident. A complete loss of binding capacity for SPMG was then noted following double mutation of both KKR and the basic domain. These findings collectively support the idea that the Tat protein offers two binding sites for SPMG. One is the wellrecognized basic domain, and the other is the newly identified
KKR region. This model is congruent with our simulationbased predictions. Notably, the mutants with either a single or a double substitution of KKR residues did not dramatically affect SPMG binding affinity, while a triple mutation significantly did. This finding highlights the importance of all three residues in this triad for functionality.

By kinetic analysis, the dissociation constant ( $K_{\mathrm{D}}$ value) was calculated as $26.5 \mathrm{pmol} / \mathrm{L}$, which represents an extremely high-affinity binding interaction (about 500 times that of the basic domain). The disparity in binding affinity between the KKR and the basic domain, in principle, should allow SPMG to preferentially occupy KKR. In silico simulation clearly demonstrated that SPMG could specifically recognize the KKR region when the disaccharide probe was used. As the length of the oligosaccharide increased, the oligomer stretched toward and along the basic domain; it began to form interactions with Arg56 when the tetramer probe was used and reached Arg53 as the sugar length increased to 8 . Ideally, SPMG at its typical length would simultaneously bind to both KKR and the whole basic domain.

An interesting feature of the KKR domain is its specificity 
for SPMG, as evident from the observation that only heparin/ HS but not other GAGs, including CSA, CSC and HA, were able to produce a dose-response-based competitive inhibitory effect on Tat-SPMG interactions. Sugar-protein interactions are predominantly dependent on saccharide composition and the extent and distribution of sulfation of the sugar backbone ${ }^{[25,26]}$. SPMG is a heparin-like sulfated polysaccharide and is rich in 1, 4-linked $\beta$ - $D$-mannuronate. The high affinity of Tat for SPMG might be the outcome of both its defined sulfate groups and its specific saccharide composition. In addition, unlike the linearly contiguous sequence of the basic domain, the KKR residues of the novel SPMG binding site, although distant in sequence, are brought into close spatial proximity via Tat conformational folding. This finding is highly consistent with current views, which hold that heparin binding sites are commonly recognized on the external surface of proteins and correspond to shallow pockets of positive charge that are often linearly contiguous or, alternatively, may be distant in sequence yet closely spatially orientated when the protein assumes its native tertiary structure ${ }^{[27-29]}$.

The KKR region facilitates Tat-mediated SLK cell adhesion in a cell surface HSPG-dependent manner ${ }^{[14]}$. In the present study, we found that SPMG dramatically inhibited SLK adhesion induced by Tat and KKR-dominant Tat (GST-Tat ${ }_{\left(G 48-\text { R57) }_{\mathrm{A}}\right)}$ as well, suggesting the interaction of SPMG with the KKR region competitively abrogated Tat-mediated cell adhesion.

In sum, we herein show that a domain consisting of a triad of Lys12, Lys41, and Arg78 is a novel, high-affinity, spatially enclosed SPMG binding site on Tat. This finding, together with the fact that SPMG inhibits Tat-induced AIDS-KS, provides important new insights into the molecular mechanism of SPMG and a potential therapeutic intervention for Tat-related Kaposi's sarcoma.

\section{Acknowledgements}

This work was supported by the National Natural Science Foundation of China (№ 30772884).

\section{Author contribution}

Han-dong JIANG and Xin-liang XIN conceived and designed the experiments. Yan-lin WU, Jing AI, Xin-liang XIN, Jingming ZHAO, and Xiao-jie XIN performed the experiments. Yan-lin WU, Jing AI, Mei-yu GENG, Han-dong JIANG, and Xin-liang XIN analyzed the data. Han-dong JIANG and Jing AI wrote the paper. And Bing XIONG designed and executed molecular docking.

\section{References}

1 Dezube BJ. Management of AIDS-related Kaposi's sarcoma: advances in target discovery and treatment. Expert Rev Anticancer Ther 2002; 2: 193-200.

2 Aversa SM, Cattelan AM, Salvagno L, Crivellari G, Banna G, Trevenzoli $\mathrm{M}$, et al. Treatments of AIDS-related Kaposi's sarcoma. Crit Rev Oncol Hematol 2005; 53: 253-65.

3 Sullivan RJ, Pantanowitz L, Dezube BJ. Targeted therapy for Kaposi sarcoma. BioDrugs 2009; 23: 69-75.
4 Aoki Y, Tosato G. HIV-1 Tat enhances Kaposi sarcoma-associated herpesvirus (KSHV) infectivity. Blood 2004; 104: 810-4.

5 Pati S, Foulke JS Jr, Barabitskaya O, Kim J, Nair BC, Hone D, et al. Human herpesvirus 8-encoded VGPCR activates nuclear factor of activated T cells and collaborates with human immunodeficiency virus type 1 Tat. J Virol 2003; 77: 5759-73.

6 Barillari G, Sgadari C, Fiorelli V, Samaniego F, Colombini S, Manzari $\mathrm{V}$, et al. The Tat protein of human immunodeficiency virus type1 promotes vascular cell growth and locomotion by engaging the alpha5beta 1 and alphavbeta 3 integrins and by mobilizing sequestered basic fibroblast growth factor. Blood 1999; 94: 663-72.

7 Rusnati M, Presta M. HIV-1 Tat protein and endothelium: from protein/cell interaction to AIDS-associated pathologies. Angiogenesis 2002; 5: 141-51.

8 Ziegler A, Seelig J. Interaction of the protein transduction domain of HIV-1 TAT with heparan sulfate: binding mechanism and thermodynamic parameters. Biophys J 2004; 86: 254-63.

9 Rusnati M, Coltrini D, Oreste P, Zoppetti G, Albini A, Noonan D, et al. Interaction of HIV-1 Tat protein with heparin. Role of the backbone structure, sulfation, and size. J Biol Chem 1997; 272: 11313-20.

10 Rusnati M, Tulipano G, Spillmann D, Tanghetti E, Oreste P, Zoppetti $\mathrm{G}$, et al. Multiple interactions of HIV-I Tat protein with size-defined heparin oligosaccharides. J Biol Chem 1999; 274: 28198-205.

11 Rusnati M, Tulipano G, Urbinati C, Tanghetti E, Giuliani R, Giacca $\mathrm{M}$, et al. The basic domain in HIV-1 Tat protein as a target for polysulfonated heparin-mimicking extracellular Tat antagonists. J Biol Chem 1998; 273: $16027-37$.

12 Tyagi M, Rusnati M, Presta M, Giacca M. Internalization of HIV-1 tat requires cell surface heparan sulfate proteoglycans. J Biol Chem 2001; 276: 3254-61.

13 Fittipaldi A, Giacca M. Transcellular protein transduction using the Tat protein of HIV-1. Adv Drug Deliv Rev 2005; 57: 597-608.

14 Ai J, Xin X, Zheng M, Wang S, Peng S, Li J, et al. A triad of Lys12, Lys41, Arg78 spatial domain, a novel identified heparin binding site on Tat protein, facilitates Tat-driven cell adhesion. PLoS One 2008; 3 : e2662.

15 Rusnati M, Urbinati C, Caputo A, Possati L, Lortat-Jacob H, Giacca M, et al. Pentosan polysulfate as an inhibitor of extracellular HIV-1 Tat. J Biol Chem 2001; 276: 22420-5.

16 Hui B, Xia W, Li J, Wang L, Ai J, Geng M. Sulfated polymannuroguluronate, a novel anti-acquired immune deficiency syndrome drug candidate, blocks neuroinflammatory signalling by targeting the transactivator of transcription (Tat) protein. J Neurochem 2006; 97: 334-44.

17 Lu CX, Li J, Sun YX, Qi X, Wang QJ, Xin XL, et al. Sulfated polymannuroguluronate, a novel anti-AIDS drug candidate, inhibits HIV-1 Tatinduced angiogenesis in Kaposi's sarcoma cells. Biochem Pharmacol 2007; 74: 1330-9.

18 Peloponese JM Jr, Gregoire C, Opi S, Esquieu D, Sturgis J, Lebrun E, et al. ${ }^{1} \mathrm{H}^{13} \mathrm{C}$ nuclear magnetic resonance assignment and structural characterization of HIV-1 Tat protein. C R Acad Sci III 2000; 323: 883-94.

19 Sali A. Comparative protein modeling by satisfaction of spatial restraints. Mol Med Today 1995; 1: 270-7.

20 Trott O, Olson AJ. AutoDock Vina: improving the speed and accuracy of docking with a new scoring function, efficient optimization, and multithreading. J Comput Chem 2010; 31: 455-61.

21 DeLano WL. The PyMOL Molecular Graphics System. 2002. Available from: http://www.pymol.org.

22 Li FC, Qi X, Geng MY. A novel method of Immobilization of 911 for surface plasmon resonance biosensor. Chin J Anal Chem 2003; 31: 
266-9.

23 Liu H, Geng M, Xin X, Li F, Zhang Z, Li J, et al. Multiple and multivalent interactions of novel anti-AIDS drug candidates, sulfated polymannuronate (SPMG)-derived oligosaccharides, with gp120 and their antiHIV activities. Glycobiology 2005; 15: 501-10.

24 Hutchings $\mathrm{H}$, Ortega N, Plouet J. Extracellular matrix-bound vascular endothelial growth factor promotes endothelial cell adhesion, migration, and survival through integrin ligation. FASEB J 2003; 17: 15202.

25 Mulloy B. The specificity of interactions between proteins and sulfated polysaccharides. An Acad Bras Cienc 2005; 77: 651-64.
26 Katsuraya K, Nakashima H, Yamamoto N, Uryu T. Synthesis of sulfated oligosaccharide glycosides having high anti-HIV activity and the relationship between activity and chemical structure. Carbohydr Res 1999; 315: 234-42.

27 Capila I, Linhardt RJ. Heparin-protein interactions. Angew Chem Int Ed Engl 2002; 41: 391-412.

28 Hileman RE, Fromm JR, Weiler JM, Linhardt RJ. Glycosaminoglycanprotein interactions: definition of consensus sites in glycosaminoglycan binding proteins. Bioessays 1998; 20: 156-67.

29 Munoz EM, Linhardt RJ. Heparin-binding domains in vascular biology. Arterioscler Thromb Vasc Biol 2004; 24: 1549-57. 